\title{
Crystallization of Liquid Water in a Molecular Dynamics Simulation
}

\author{
Igor M. Svishchev and Peter G. Kusalik \\ Department of Chemistry, Dalhousie University, Halifax, Nova Scotia, Canada B3H 4J3
}

(Received 16 May 1994)

\begin{abstract}
In this Letter we report our success in crystallizing a bulk sample of liquid water in molecular dynamics simulations. In these computer experiments supercooled liquid TIP4P water at $250 \mathrm{~K}$ was subjected to a homogeneous static electric field; the resulting polar crystal which forms within 200 ps has the structure of ice $I_{c}$. These simulation results suggest that the local electric fields that exist near the surfaces of various materials or within confined geometries can play an important role in promoting the crystallization of liquid water.
\end{abstract}

PACS numbers: 61.25.Em, 64.60.Qb, 64.70.Dv

\begin{abstract}
Although the structure and physical properties of liquid water at room temperature [1-3] are now well established, a fundamental understanding of its lowtemperature behavior [4-6] is still lacking. Upon freezing, water can form various crystalline and amorphous ices [2,4-6]. Under certain conditions these solids exhibit a complex series of phase transformations [4-8] which, together with the thermodynamic anomalies of the supercooled liquid $[4,6]$, remain the subject of intense debate. In recent years computer simulations have contributed greatly to this area. Among many such studies [6,8-13] the enormous computational efforts
\end{abstract} undertaken by Poole, Sciortino, Essmann, and Stanley $[6,8]$ to model metastable phases of solid water are of particular note. In their work they have used molecular dynamics (MD) simulations to help construct a phase diagram for low- and high-density amorphous ice. However, despite these tremendous computational efforts, no one had yet succeeded in crystallizing a bulk sample of water in a computer simulation. We are aware of no simulation study in which any isotropic molecular liquid was continuously transformed into a regular solid. It is not surprising then that freezing phenomena [14], although among the most fundamental in nature, still remain rather poorly understood at the microscopic level.

Thermodynamics views the transformation of a molecular liquid into a molecular crystal as a first-order phase transition. In the absence of a foreign surface or particle to promote heterogeneous nucleation, this phase transition begins with the spontaneous nucleation of molecules into small aggregates which, if they reach a critical size, form the seeds for the new phase. Unfortunately, computer simulation methods have been unable to model this dynamical process directly, the large-scale density and polarization fluctuations that might initiate formation of a heterophase have not been recorded even for very large systems. Upon reducing the temperature, phase trajectories tend to get trapped into a region of metastable glassy states [6] which makes it impossible to follow further the evolution of a molecular system towards its crystalline state.
We have attempted to bypass these limitations by applying a static electric field in MD simulations of TIP4P [15] water and have discovered that the deeply supercooled liquid can be completely transformed into a polar crystal with the regular structure of ice I within only a few hundred picoseconds. The field strengths we have considered, 0.1 and $0.5 \mathrm{~V} / \AA$, are smaller than the average local (internal) electric fields within condensed phases of water (which range around 1.5-2.0 V/ $\AA$ ). These values also lie within the operating range of modern lasers [16] and are comparable with the local electric fields experienced by water molecules near the surfaces of biopolymers [17] or within the cracks of amino acid crystals [18]. In this Letter we describe our computer experiments and present our findings.

Our MD simulations of TIP4P water were performed with samples of 256 molecules at the density of $0.95 \mathrm{~g} / \mathrm{cm}^{3}$. This density roughly corresponds to that of a stable TIP4P ice $\mathrm{I}_{h}$ crystal at $270 \mathrm{~K}$ [10]. As demonstrated in earlier studies the TIP4P potential is reasonably successful in describing the properties for water [15] and for various amorphous $[6,8]$ and crystalline ices $[10,19]$. Ewald sums [20] were utilized in periodic boundary conditions with a truncated octahedral simulation cell [21]. Our implementation of the Ewald method and other details of our simulation methodology are described in Ref. [22]. The temperature was controlled throughout with the aid of a Gaussian thermostat [23]. The homogeneous static electric field was always applied along the laboratory $Z$ axis.

An initial bulk sample of supercooled liquid water was prepared by taking a well-equilibrated configuration of TIP4P water at $298 \mathrm{~K}$ and slowly cooling it to $250 \mathrm{~K}$. The resulting configuration was equilibrated for a further $200 \mathrm{ps}$ followed by a 150 ps production run during which estimates for the average total configuration energy, the pressure, the dielectric constant, and the translational self-diffusion coefficient were collected. The values obtained, $\langle U\rangle=-45.1 \mathrm{~kJ} / \mathrm{mol}, P=-109 \mathrm{MPa}$, $\varepsilon=56$, and $D=0.69 \times 10^{-5} \mathrm{~cm}^{2} / \mathrm{s}$, respectively, agree well with previous estimates obtained by Poole et al. $[6,8]$ 
for the same state point and by Ruocco et al. [9] for a nearby state point. The radial distribution function (RDF) of oxygen atoms, $g_{0 O}(r)$, for liquid TIP4P water at $250 \mathrm{~K}$ is displayed in Fig. 1(a) and matches the data reported in Ref. [8].

A static electric field was then applied to this liquid system. For a field strength of $0.5 \mathrm{~V} / \AA$ at a constant temperature of $250 \mathrm{~K}$ the liquid was transformed within $200 \mathrm{ps}$ into a regular solid structure with $\langle U\rangle=$ $-49.2 \mathrm{~kJ} / \mathrm{mol}$ and $P=-176 \mathrm{MPa}$. The oxygen-oxygen RDF for this solid (accumulated over a subsequent 30 ps run) is also plotted in Fig. 1(a). This function exhibits long-range intermolecular correlations that strongly suggest a thermally agitated crystalline lattice of ice I [4]. Further insights into this structural transformation can be drawn from Fig. 2 which compares the spatial distribution function (SDF) of oxygen atoms, $g_{\mathrm{OO}}(r, \underline{\Omega})$, for the supercooled liquid [Fig. 2(a)] with that of the solid formed after the field was applied [Fig. 2(b)]. We recall that the SDF represents the spatial analog of the RDF, where $\underline{\Omega}=(\theta, \phi)$ represents the angular coordinates of the separation vector. This function completely characterizes the three-dimensional local structure in molecular systems $[3,24]$. Only $\mathrm{O} \cdots \mathrm{O}$ correlations essentially
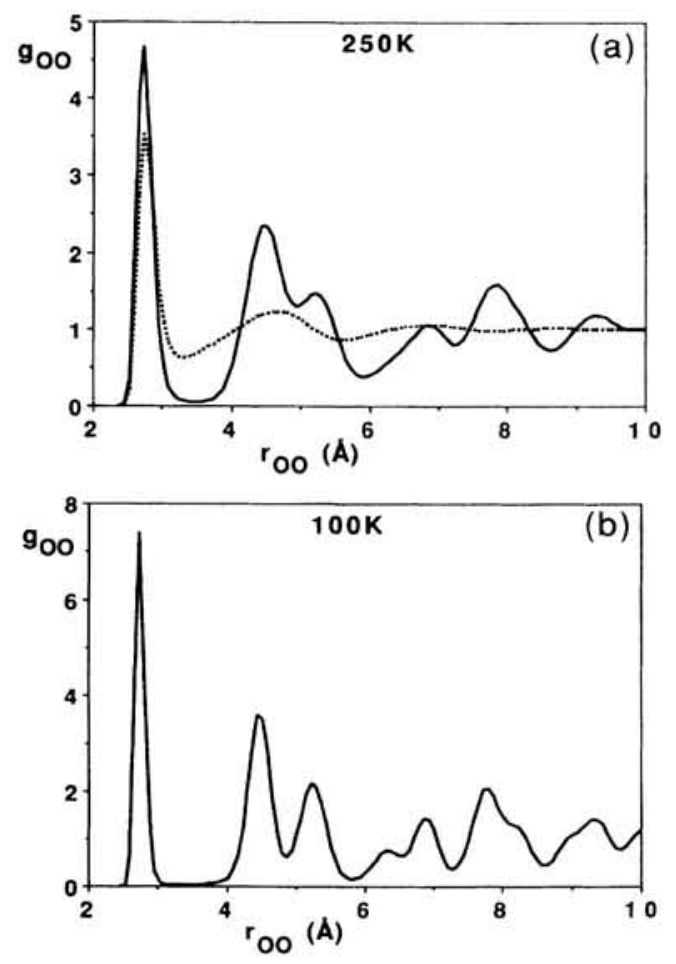

FIG. 1. The radial distribution functions of oxygen atoms in TIP4P water. (a) The supercooled liquid (dotted line) at $250 \mathrm{~K}$ with no applied field, and the polar ice (solid line) formed from this liquid at $250 \mathrm{~K}$ under the field of $0.5 \mathrm{~V} / \AA$. (b) Polar ice I at $100 \mathrm{~K}$.
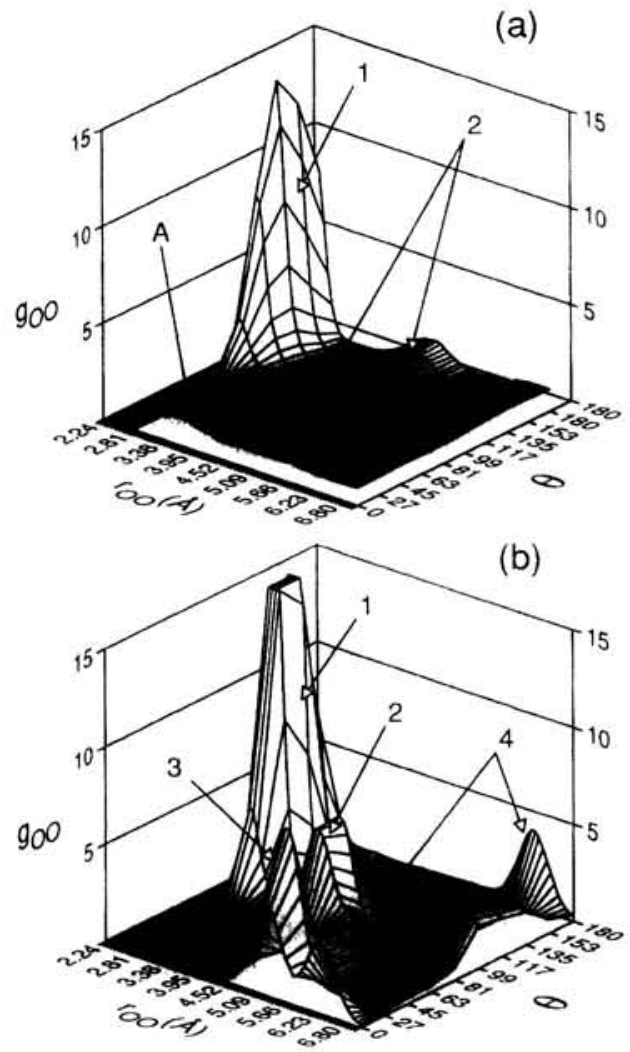

FIG. 2. The spatial distribution function of oxygen atoms in TIP4P water at $250 \mathrm{~K}$. (a) The supercooled liquid with no applied field and (b) the polar ice formed under a field of $0.5 \mathrm{~V} / \AA$. $\theta$ denotes the angle between the dipole axis of the central water molecule and the oxygen-oxygen separation vector. 1, 2, 3, and 4 identify, respectively, the first, the second, the third, and the fourth tetrahedrally bonded neighbors of the continuous H-bond network, and $A$ represents additional nontetrahedral coordination. In (b) the nearest-neighbor peak (labeled 1) was truncated, the actual height being about 39 .

perpendicular to the plane of the central molecule (corresponding to $\phi=90$ in Ref. [3]) are plotted in Fig. 2.

Figure 2 provides direct evidence that the interstitial nontetrahedral coordination in liquid water [3], a local maximum in $g_{\mathrm{Oo}}(r, \underline{\Omega})$ at approximately $3.3-3.9 \AA$ [see Fig. 2(a)], disappears when the supercooled liquid transforms into an ice [see Fig. 2(b)]. These interstitially coordinated molecules in water structure must clearly play a crucial role in characterizing the liquid water to ice transition. Another distinctive structural change evident from the spatial distributions of oxygen atoms is associated with the second (H-bonded) neighbors (identified as 2 in Fig. 2). In the supercooled liquid these neighbors appear over a wide range of orientations in the local frame, as can be easily seen in Fig. 2(a). During the phase transition they collapse onto distinctive sites in the local frame giving rise to a sharp pair-density maximum in the SDF [see Fig. 2(b)]. Moreover, the third and fourth 
(H-bonded) neighbors which are virtually undetectable in the liquid water SDF [Fig. 2(a)] appear as well-defined maxima in Fig. 2(b) (labeled as 3 and 4). We remark that for our crystalline solid the SDF representing $\mathrm{O} \cdots \mathrm{O}$ correlations exhibits tetrahedral symmetry; for example, the function $g_{\mathrm{OO}}(r, \underline{\Omega})$ in the plane of the central molecule (i.e., $\phi=0$ ) is equivalent to the out-of-plane result shown in Fig. 2(b). This is not true in the liquid state, as demonstrated in Fig. 3 of Ref. [3].

A second independent 200 ps run (starting from a different liquid configuration) was performed with a field of $0.5 \mathrm{~V} / \AA$ and virtually identical results were obtained. We have also carried out a third 200 ps simulation in which the supercooled TIP4P liquid was subjected to a field of $0.1 \mathrm{~V} / \AA$. No dramatic changes were detected in the liquid structure to suggest the system was again undergoing a phase transition. These observations imply that for the current state point the threshold value of the electric field strength which will induce TIP4P water to undergo a liquid to solid phase transition lies between 0.1 and $0.5 \mathrm{~V} / \AA$.

In order to gain a more complete microscopic understanding of this field-induced phase transition we have visualized specific molecular configurations generated during our MD simulation. Several of these are shown in Fig. 3 as viewed down the electric field vector (the $Z$ axis). Figure 3(a) portrays the structure in supercooled liquid water in the absence of the electric field as a

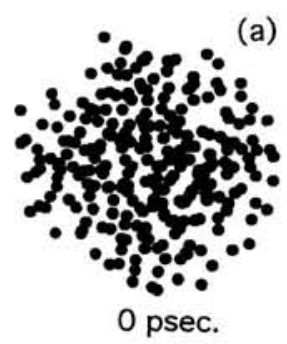

(c)

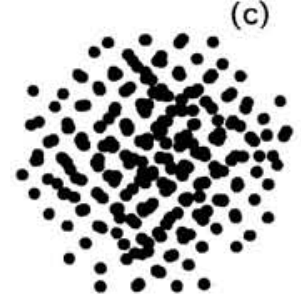

150 psec.

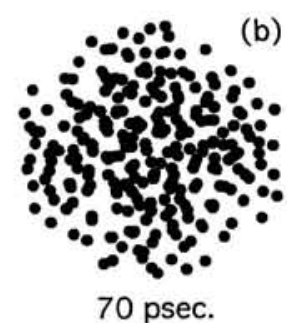

(d)

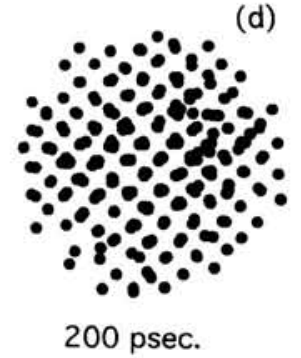

FIG. 3. Molecular configurations of a water system generated during a field-induced phase transition. (a) Initial liquid water configuration. (b), (c), and (d) Applied-field results at different stages of ice formation. The dots represent the oxygen atoms of the water molecules projected onto the $x-y$ plane of the laboratory-fixed frame. The applied field is acting along the normal to the plane of the figure. All data are at $250 \mathrm{~K}$ for a field of $0.5 \mathrm{~V} / \AA$. random distribution of instantaneous particle positions. Figure 3(b) depicts the molecular configuration $70 \mathrm{ps}$ after the field was applied and comparison with Fig. 3(a) reveals that some structural ordering has already taken place in the system. A more detailed inspection of this configuration at many different orientations reveals some clustering and layering within the system somewhat analogous to the structure we have observed in lowdensity amorphous ice [25]. As the system continues to evolve under the applied field conditions, further clustering becomes apparent and molecules begin to occupy regular lattice sites; this process can be clearly seen in Fig. 3(c). At 200 ps [Fig. 3(d)] almost all the water molecules occupy regular positions in a periodic structure.

The still imperfect ice crystal that emerges from the supercooled liquid upon application of the electric field melts in the absence of the field at $250 \mathrm{~K}$. Reducing the temperature to $200 \mathrm{~K}$ we obtain crystalline samples that appear mechanically stable in the absence of the electric field. This temperature dependence is in accord with the work of Haymet and co-workers [10] where a stable solidliquid interface for TIP4P water required a temperature of $240 \mathrm{~K}$. The local structure in one of these samples at $100 \mathrm{~K}$ is represented by the oxygen-oxygen RDF plotted in Fig. 1(b). Not surprisingly, this function appears very similar to experimental $\mathrm{x}$-ray diffraction curves from lowtemperature crystals of ice I $[4,26]$.

A critical question to address at this point is what modification of ice I, cubic or hexagonal, have we formed from the supercooled liquid? Figure 4 displays several crystallographic planes for the low-temperature $(100 \mathrm{~K})$ ice sample whose RDF is shown in Fig. 1(b); the solid

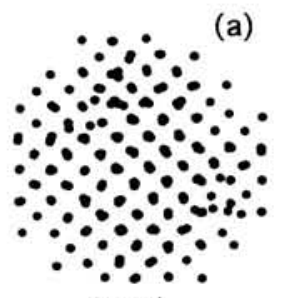

$(001)$

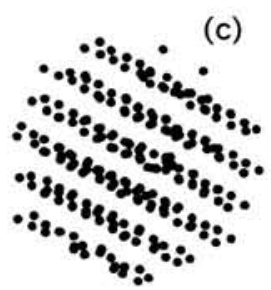

(123)

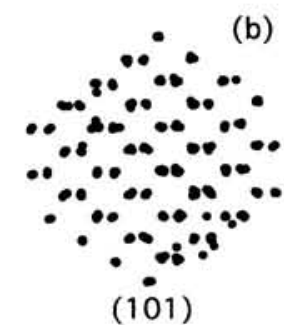

(d)

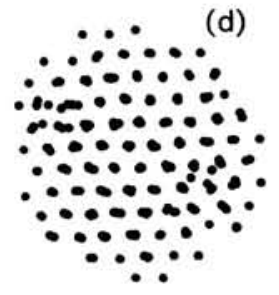

(111)
FIG. 4. Crystallographic planes for cubic TIP4P ice formed from the supercooled liquid under an applied electric field. All data are at $100 \mathrm{~K}$. 
can be unambiguously identified as the cubic phase. For example, the (101) plane of the crystal shown in Fig. 4(b) indicates that as in cubic ice I [2,5] the open "shafts" formed by the hexagonal rings of this tetrahedral lattice are not perpendicular to the plane of the rings (in the hexagonal ice I $[2,5]$ these open "shafts" run parallel to the $c$-crystal axis and are perpendicular to the plane of the rings). To provide additional evidence careful visualization of a configuration of this crystal demonstrates that the puckering layers of hexagonal rings [see Fig. 4(c)] are packed in such a way that three neighboring oxygen atoms in one layer and the three neighboring oxygen atoms in the adjacent layer have a "chair" conformation, an arrangement found only in the cubic modification of ice $I$. In ice $I_{h}$ the adjacent layers are joined exclusively with the hexagonal rings in the "boat" conformation [2].

Liquid water under normal conditions freezes into hexagonal ice. Cubic ice can be formed either by condensing water vapor on a cold surface or by warming amorphous ice or a high-pressure crystalline polymorph $[2,5]$. One explanation for why the electric field in our computer experiment induces the formation of ice $I_{c}$ rather than of ice $I_{h}$ from the supercooled liquid is that the diamond-type packing of cubic ice, unlike the isomorphous hexagonal packing, can support an ideal parallel arrangement of the molecular dipoles. The ice crystal displayed in Fig. 4 is a polar substance and obviously the relatively large applied field drives the system towards its most favorable "in-field" configuration. The dipolar saturation in this system is almost $90 \%$, a value that would be unattainable with an ice $\mathrm{I}_{h}$ crystal.

Combinations of various theoretical and experimental methods are certainly needed to provide further insights into field-induced transition phenomena of low-temperature water. Aside from their fundamental theoretical interest, these studies promise new advances in areas such as material design (e.g., clathrate formation). The present results suggest that the local electric fields which occur at the surfaces of various materials can play an important role in promoting the crystallization of liquid water. In a recent experiment study of ice nucleation on the surfaces of hydrophobic amino acid crystals that have no structural match with ice, Gavish et al. [18] hypothesized the crucial role played by large electric fields arising within substrate cracks. Our simulation results strongly favor these speculations. We conclude this Letter by adding that a forthcoming paper will focus specifically upon the field-induced transition of supercooled water into amorphous ice. Work is also underway to explore pressure (density) and polarization dependences in the freezing of water.
We are grateful for the financial support of the Natural Sciences and Engineering Research Council of Canada.

[1] Water-A Comprehensive Treatise, edited by F. Franks (Plenum, New York, 1972), Vol. 1.

[2] D. Eisenberg and W. Kauzmann, The Structure and Properties of Water (Oxford Univ. Press, Oxford, 1969).

[3] I. M. Svishchev and P.G. Kusalik, J. Chem. Phys. 99. 3049 (1993).

[4] Water-A Comprehensive Treatise (Ref. [1]), Vol. 7.

[5] P. V. Hobbs, Ice Physics (Clarendon, Oxford, 1974).

[6] P. H. Poole, F. Sciortino, U. Essmann, and H. E. Stanley, Nature (London) 360, 324 (1992).

[7] O. Mishima, J. Chem. Phys. 100, 5910 (1994).

[8] P. H. Poole, F. Sciortino, U. Essmann, and H. E. Stanley, Phys. Rev. B 48, 4605 (1993).

[9] G. Ruocco, M. Sampoli, A. Torcini, and R. Vallauri, J. Chem. Phys. 99, 8095 (1993).

[10] B. B. Laird and A. D. J. Haymet, Chem. Rev. 92, 1819 (1992); O. A. Karim and A. D. J. Haymet, J. Chem. Phys. 89, 6889 (1988).

[11] C. Lee, D. Vanderbilt, K. Laasonen, R. Car, and M. Parrinello, Phys. Rev. B 47, 4863 (1993).

[12] J.S. Tse and M.L. Klein, Phys. Rev. Lett. 58, 1672 (1987).

[13] H. T. Taylor and B. N. Hale, Phys. Rev. B 47, 9732 (1993).

[14] H. Lowen, Phys. Rep. 237, 249 (1994).

[15] W. L. Jorgensen, J. Chandrasekhar, J. D. Madura, R. W. Impey, and M. L. Klein, J. Chem. Phys. 79, 926 (1983).

[16] S.-B. Zhu, S. Singh, and G.W. Robinson, Adv. Chem. Phys. 85, 627 (1994).

[17] W. Drost-Hansen and J. Lin Singleton, Fundamentals of Medical Cell Biology (JAI Press, Greenwich, CT, 1992), Vol. 3a, p. 157.

[18] M. Gavish, J.L. Wang, M. Eisenstein, M. Lahav, and M. Leisterowitz, Science 256, 815 (1992).

[19] J. S. Tse and M. L. Klein, J. Phys. Chem. 92, 315 (1988); J. S. Tse, M. L. Klein, and I. R. McDonald, J. Chem. Phys. 81, 6124 (1984).

[20] M. P. Allen and D. J. Tildesley, Computer Simulations of Liquids (Oxford Univ. Press, Oxford, 1987).

[21] D. Fincham and D. M. Heyes, Adv. Chem. Phys. 63, 493 (1985).

[22] I. M. Svishchev and P. G. Kusalik, J. Phys. Chem. 98, 728 (1994).

[23] D. J. Evans and G.P. Morriss, Statistical Mechanics of Nonequilibrium Liquids (Academic, San Diego, 1990).

[24] I. M. Svishchev and P. G. Kusalik, J. Chem. Phys. 100, 5165 (1994).

[25] I. M. Svishchev and P. G. Kusalik (to be published).

[26] A. H. Narten, C. G. Venkatesh, and S. A. Rice, J. Chem. Phys. 64, 1106 (1976). 

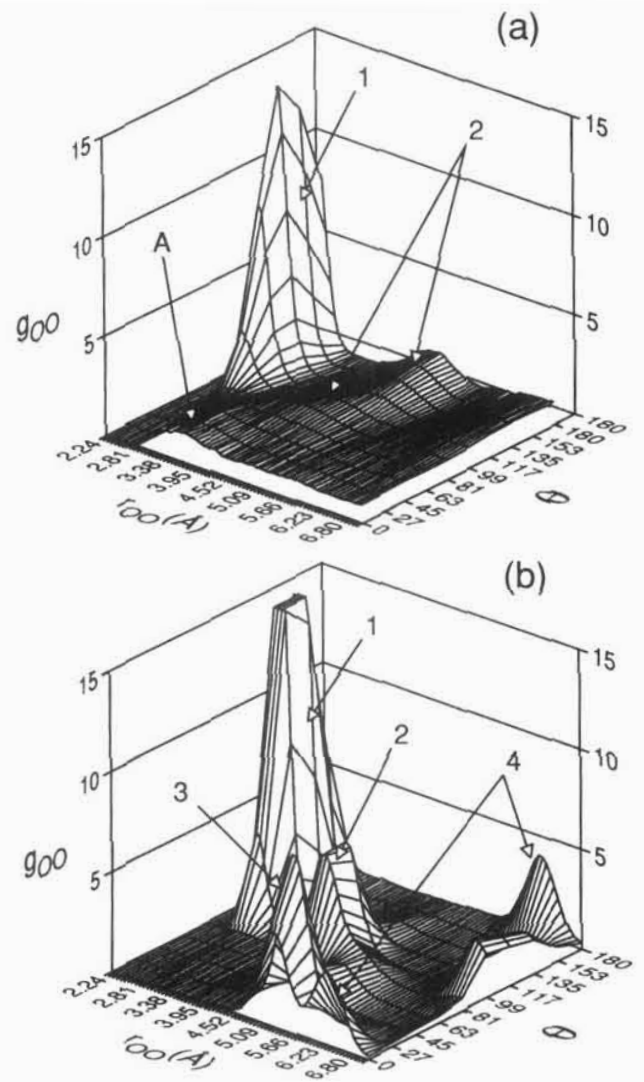

FIG. 2. The spatial distribution function of oxygen atoms in TIP4P water at $250 \mathrm{~K}$. (a) The supercooled liquid with no applied field and (b) the polar ice formed under a field of $0.5 \mathrm{~V} / \AA$. $\theta$ denotes the angle between the dipole axis of the central water molecule and the oxygen-oxygen separation vector. 1, 2, 3, and 4 identify, respectively, the first, the second, the third, and the fourth tetrahedrally bonded neighbors of the continuous $\mathrm{H}$-bond network, and $A$ represents additional nontetrahedral coordination. In (b) the nearest-neighbor peak (labeled 1) was truncated, the actual height being about 39. 